Article

\title{
Life and Death of Industrial Ecosystems
}

\author{
Weslynne S. Ashton ${ }^{1, *}$, Shauhrat S. Chopra ${ }^{2}$ and Rahul Kashyap ${ }^{3}$ \\ 1 Stuart School of Business, Illinois Institute of Technology, 10 West 35th Street, Chicago, IL 60616, USA \\ 2 Institute for Environmental Science and Policy, University of Illinois at Chicago, \\ 2121 West Taylor Street (MC 673), Chicago, IL 60612-4224, USA; chopras@uic.edu \\ 3 Department of Physics, University of Buffalo, Fronczak Hall, Buffalo, NY 14260-1500, USA; \\ rahulkas@buffalo.edu \\ * Correspondence: washton@iit.edu; Tel.: +1-312-906-6517
}

Academic Editor: Marc A. Rosen

Received: 9 February 2017; Accepted: 7 April 2017; Published: 13 April 2017

\begin{abstract}
Self-organized industrial ecosystems (SOIEs) refer to communities of firms in diverse industries that spontaneously engage in Industrial Symbiosis (IS); that is, firms independently develop bilateral and multi-lateral interactions involving material, energy, and knowledge sharing for individual and collective benefit. Like biological ecosystems, self-organized industrial ecosystems must constantly respond to external perturbations. Resilience of SOIEs, or the ability of systems to maintain structure and function in response to perturbations, has been the focus of a few recent studies. However, these studies have only examined the network characteristics for resilience of IS in a static manner. The current study contributes to this emerging literature by examining the dynamics associated with growth (life) and demise (death) of self-organized industrial ecosystems in light of changing network dynamics and external perturbations, with emphasis on material and socio-economic aspects of connectivity between firms. This research is grounded in real world cases, but expands beyond these through hypothetical network models in order to ascertain the network characteristics that lead to more resilient structures and outcomes. A key distinction is made between SOIEs that include an anchor firm versus scavenger firms. The former typically involve a scale-free network structure where new member firms preferentially connect to actors with the most connections, while the latter involve more random, fully-connected networks where new member firms connect with multiple existing actors. The results imply that resilience of SOIEs do not arise from intrinsic properties of the system alone, but from the interplay of network topology with external social and ecological constraints.
\end{abstract}

Keywords: industrial symbiosis; resilience; sustainability, network dynamics

\section{Introduction}

Industrial Symbiosis (IS) describes the phenomenon whereby a group of firms that do not traditionally have relationships with each other collaborate to more effectively manage their resources, particularly by-products or utilities, and in so doing realize public and private economic, environmental, and social benefits. IS has grown tremendously as an area of study since the early 1990s when the first instance of IS, in Kalundborg, Denmark, gained international recognition [1]. Kalundborg is a complex and continuously evolving network (or industrial ecosystem) of firms in different industries whose collaboration on energy, water, material, and waste resource management resulted in economic, environmental, and social benefits for the individual firms and town as a whole [2].

Numerous projects around the world have since sought to promote IS either through identifying and stimulating existing industrial ecosystems and/or planning and developing Eco-Industrial Parks (EIPs) or Eco-Industrial Networks (EINs), more broadly [3]. Boons and colleagues point out that as 
a result of these two different approaches, academic studies of IS include phenomena that vary in goals, drivers, network configuration, and outcomes, which has made the comparative study of IS across the world problematic [4]. As such, they suggest that distinctions be made among EINs with seven different "dynamics" or processes of development. Self-organization is the dynamic most associated with an organic or unplanned approach, in which actors develop bilateral symbiotic relationships in the absence of collective goals [4]. The typical pattern of development of a self-organized industrial ecosystem (SOIE) follows: a few firms develop bilateral by-product synergies for individual benefit and are unaware of being a part of a larger system of synergies; the complete network of firms involved in synergies is later uncovered, which makes actors aware of being part of this larger system; existing firms and other interested stakeholders actively work to attract new firms to the system, grow the number of synergies, and collaborate towards increasing overall sustainability. The organizational boundary change dynamic has some elements of self-organization as a single firm organically develops internal symbiosis among different business units and later spins these out into separate businesses. In the other five dynamics, actors purposefully collaborate to develop symbiotic ties among firms and collectively improve economic and environmental performance. While both of the former are primarily driven by firms, the other five are driven by government or third-party actors [4]. As such, self-organization is viewed as very distinct from the facilitation and planning approaches.

Failed Industrial Ecosystems: There are only a few examples of failed SOIEs or EINs in the literature. Many of these were proposed EINs that never truly materialized [3,5], which fit into Boons and colleagues' pilot facilitation or government planning dynamics [4]. The first published example of failed self-organized industrial symbiosis was in Barceloneta, Puerto Rico, where firms primarily in the pharmaceutical sector collaborated through water and material synergies for decades but IS in the network collapsed in the mid-2000s when most of the firms in that sector ceased operations on the island [6]. The demise of the network occurred because of pressures and mergers on the global pharmaceutical industry as well as a regional economic policy removing tax breaks that had been a major boon for manufacturers on the island. The scale of external economic perturbations crippled the island's economy and destroyed the relevance of symbiosis for the majority of the firms that were going out of business. Ashton hypothesized that industrial ecosystems develop through several successional phases much like other complex adaptive systems, including exploitation, conservation, release, and reorganization as described in Holling's panarchy framework, which aligns with ideas of ecological resilience [6,7].

Complexity, Resilience, and Sustainability in Industrial Ecosystems: The resilience of self-organized industrial ecosystems, that is, how these networks respond to and resist change, has been the focus of a few recent studies [8-11]. Resilience in industrial ecosystems has been defined as the capability of a system to absorb disruptions while maintaining its structure and function [9]. Zhu and Ruth interpreted resilience as maintaining "eco-efficient material and energy flows" in the face of disruptions that include changes in "production levels and technologies, and firm closure and relocation" [8]. It is an emergent property in self-organized systems, which can nonetheless be strategically designed in SOIEs and other EINs through redundant and multifunctional linkages. Most self-organized industrial ecosystems have linkages that are coincidental rather than strategic, and thus may be more vulnerable to perturbations. Similarly, many EINs are built around anchors to which many other actors are connected; these more central nodes are of greater critical importance for network resilience and stability [9]. The role of various network structures on resilience has only recently been examined for a few successful cases of SOIEs mostly because of a paucity of data. However, a thorough examination of network structures for distinct SOIE growth dynamics, including failed systems, is necessary to understand their implications for the development of resilient industrial symbiosis systems. This article contributes to this emerging literature by examining the dynamics associated with growth and demise of SOIEs in light of changing network dynamics and external perturbations, with emphasis on the material (typically by-product exchanges or utility sharing) and socio-economic (social and economic ties) aspects of connectivity between firms. 
Aims: This paper aims to understand the dynamics driving growth (life) and demise (death) of self-organized industrial ecosystems by developing hypothetical network models of the behavior of firms in SOIEs. It focuses on the self-organization dynamic as this study surmises that there are hundreds of these systems worldwide, but that most have yet to be uncovered by researchers [3]. Thus, they provide an interesting population to be modeled as resilience implies such systems have survived in the long term, whereas there may be numerous deaths of unidentified systems. This research is grounded in real-world cases but expands beyond these through hypothetical network models in order to ascertain the characteristics of the networks that lead to more resilient structures and outcomes. A key objective is to identify the network characteristics that make some networks more resilient, while others are more vulnerable to perturbations. The dynamics of SOIEs based on literature review is presented next, along with a description of the model development and discussion how the literature informed this process. This is followed by results and discussion of implications for SOIE research. This model also provides insights for establishing a resilience driven framework for stimulating the development of industrial symbiosis, which has been advocated in the literature.

\section{Materials and Methods}

Dynamics of Self-Organized Industrial Ecosystems: In order to develop realistic hypothetical models, this study first reviewed the literature to better understand two aspects of SOIE dynamics-factors that lead to the growth and demise of symbiotic linkages in networks, and the network structure of SOIEs at various stages of development, including system collapse.

Walls and Paquin describe factors that enable EINs to launch, "antecedents", help them to grow, "lubricants," prevent their growth, "limiters," as well as common results of their activities or "consequences" [12]. Their framework does not distinguish how these factors differ among different IS dynamics. As such, we have selected those factors that are relevant to self-organized industrial ecosystems only and detailed them in Table 1. Unlike in other EINs, intermediaries or government interventions do not appear to have a prominent role in SOIEs, instead the policy framework and socio-cultural norms establishes the rules, which make by-product reuse or other sharing relationships possible. Resource abundance or constraints and individual firm motives are the most important drivers for firms self-organizing into symbiotic relationships. The prevalence of anchors or scavengers is an important distinction as it determines the network structure. Anchors tend to be large industries that have abundant resources that can serve as raw materials for many partners, forming scale-free networks where new members of the network preferentially connect to actors with the most connections, emblematic of Kalundborg. On the other hand, scavengers tend to be small and specialized in recovering particular classes of materials, and many of them often co-exist in EINs, so that new members can connect with multiple existing actors, forming more of a random network structure, such as in the case of Styria. Lubricants are mainly social factors that embed the relationships among participants in the network and go beyond the material exchanges that are the hallmarks of IS. Limiters include factors internal to the network, such as diversity and power asymmetries among participants, as well as external perturbations such as regulatory changes. The consequences of IS-economic, environmental, and social benefits—appear to be more similar across different types of EINs. Applying these factors to model development, this study utilizes antecedents to stand in for growth mechanisms, while limiters are used as mechanisms that cause ties or nodes to fail. While external perturbations are not factored into Walls and Paquin's framework, evidence from Chopra and Khanna's resilience studies [9] and Ashton's work in Barceloneta [6] suggest that major external perturbations may have an important role to play in the demise of SOIEs. 
Table 1. Factors facilitating and inhibiting the growth of self-organized industrial ecosystems.

\begin{tabular}{ll}
\hline & Presence of industries that can serve as anchors (providing materials to diverse actors, \\
& e.g., power plant providing steam or waste heat) or scavengers (receiving materials \\
from diverse actors, e.g., recyclers receiving diverse waste) & Facilitative government policies, such as waste disposal pricing or preferences, rather \\
than direct intervention or planning & Natural resource abundance or scarcity \\
& Favorable firm-level economic rationale (e.g., cost reduction, efficiency, \\
& revenue streams) \\
& Social, cultural and/or institutional pressures on firms (to cooperate, conserve \\
& resources, etc.) \\
& Trust, openness and cooperation among firm personnel \\
& Strong social network ties or social capital \\
& Knowledge creation and sharing \\
& Embeddedness (cognitive, social) \\
Lubricants & Loss of key players (e.g., individual champions, firms) \\
& Too little or too much diversity (i.e., inability to match material flows) \\
& Restrictive environmental regulations \\
& Power or status asymmetries (i.e., who wants to partner with whom) \\
& Risk concerns \\
& High dependency \\
\hline Limiters & Environmental benefits (e.g., eco-efficiency in material and energy flows) \\
& Economic benefits (e.g., cost reductions, sales increases) \\
Innovation (e.g., new product development or business models) \\
Network resilience (i.e., resistance to disruption) \\
\hline
\end{tabular}

Table 2 describes the network characteristics of several existing SOIEs at different phases of development. These include Kalundborg, Denmark, at initial formation, development and maturity, Kwinana, Australia, and Styria, Austria, at maturity, and Barceloneta, Puerto Rico, at initial formation, maturity, and collapsed phases. This study reviewed published reports, including network diagrams, to quantify the number of actors (nodes), synergies between them (edges) and industries, and identify the network structure in each, in order to ground our models in the observed conditions of diverse SOIEs over time.

In the initial phase, networks are characterized by a small number of firms participating in few synergies. As the systems develop, one or more core groups may form in which synergies are concentrated (preferential attachment to well-connected nodes), often determined by the presence of anchor firms. In the mature phase, a large number of firms are involved in numerous synergies. Preferential attachment appears to be the mode of growth in networks with a large anchor firm that either supplies or receives materials from a diverse set of other industries, such as in Kalundborg. More diverse networks, or those without a single anchor, appear to grow randomly, with new firms attaching to multiple other firms with which they have material matches, such as recycling firms in Styria. It is notable that both Kwinana and Styria at maturity phases are larger than Kalundborg and Barceloneta in terms of the number of firms and synergies, suggesting that random-growth networks have the potential to grow larger than anchor-networks. In successful SOIEs, the number of firms and synergies grow and persist over time. By contrast, systems that collapse have a breakdown of synergies and loss of firms and synergies at some point in their history. It is noteworthy that collapse need not happen at the initial stages, but may occur during more mature phases. 
Table 2. Network characteristics of self-organized industrial ecosystems in different phases of development.

\begin{tabular}{|c|c|c|c|c|c|c|}
\hline $\begin{array}{l}\text { Self-Organized } \\
\text { Industrial } \\
\text { Ecosystem }\end{array}$ & Phase (Years) & $\begin{array}{c}\text { No. } \\
\text { Entities } \\
\text { (Nodes) }\end{array}$ & $\begin{array}{l}\text { No. Entities } \\
\text { Involved in } \\
\text { Symbiosis } \\
\text { (Nodes) }\end{array}$ & $\begin{array}{c}\text { No. } \\
\text { Material } \\
\text { Synergies } \\
\text { (Edges) }\end{array}$ & $\begin{array}{l}\text { No. } \\
\text { Industries } \\
\text { (Diversity) }\end{array}$ & Network Structure \\
\hline \multirow{3}{*}{ Kalundborg [2] } & $\begin{array}{l}\text { Initial formation } \\
\quad(\text { pre-1980) }\end{array}$ & 8 & 7 & 5 & 8 & $\begin{array}{l}\text { Preferential } \\
\text { attachment, } \\
\text { single core }\end{array}$ \\
\hline & $\begin{array}{c}\text { Development } \\
(1980-2000)\end{array}$ & 12 & 12 & 19 & 11 & $\begin{array}{l}\text { Preferential } \\
\text { attachment, } \\
\text { single core }\end{array}$ \\
\hline & Maturity (post-2000) & 23 & 22 & 27 & 11 & $\begin{array}{c}\text { Preferential } \\
\text { attachment, several } \\
\text { core nodes }\end{array}$ \\
\hline Kwinana [13] & Maturity ( 2008) & 52 & 37 & 47 & 11 & $\begin{array}{l}\text { Random, many } \\
\text { redundant links }\end{array}$ \\
\hline Styria [14] & Maturity ( 1997) & 39 & 39 & 44 & 14 & $\begin{array}{l}\text { Random, many } \\
\text { redundant links }\end{array}$ \\
\hline \multirow{3}{*}{ Barceloneta [6] } & $\begin{array}{l}\text { Initial formation } \\
\quad(\text { pre-1990) }\end{array}$ & 10 & 10 & 9 & 3 & $\begin{array}{l}\text { Preferential } \\
\text { attachment, } \\
\text { single core }\end{array}$ \\
\hline & Maturity ( 2005) & 20 & 14 & 20 & 7 & $\begin{array}{l}\text { Preferential } \\
\text { attachment, } \\
\text { single core }\end{array}$ \\
\hline & $\begin{array}{l}\text { Collapsed system } \\
\quad \text { (post-2005) }\end{array}$ & 8 & 9 & 0 & 3 & $\begin{array}{c}\text { No active } \\
\text { symbiosis network }\end{array}$ \\
\hline
\end{tabular}

Model Development: A novel stochastic framework was developed to understand the tradeoffs between network properties responsible for resilient SOIEs by simulating the evolution of hypothetical SOIEs. These simulations are based on observations and insights into real world SOIEs growth dynamics and network configurations (Tables 1 and 2, respectively). Most self-organized industrial ecosystems start as very small "kernels" of IS, and likely do not move beyond the "3-2" heuristic (that is, at least three firms engaged in at least two synergies) that has been used to define industrial symbiosis [3]. Such systems, because of their small size, are extremely vulnerable to external changes, such as changes in raw materials or production processes in individual firms or supply disruptions or natural or man-made disasters that affect one or more of the firms involved and cause the "kernel" to collapse or disappear. However, it is hypothesized here that once industrial ecosystems exceed a particular size threshold, they are less likely to collapse as a dense network of material and social connections increase their resistance to disturbances affecting one of more actors. The presence of strong social ties in addition to individual economic benefits may embed the value of IS among the participants [15,16], and make large scale collapse harder to occur, as the actors continuously seek collaborative resource management arrangements even after individual synergies collapse or firms move or go out of business.

The overarching framework developed here involves three stages: (1) repetitive simulation of SOIE network evolution driven by two different growth dynamics (preferential growth and random growth) under two scenarios (with and without disaster), (2) sorting of SOIE network snapshots into "dead" and "alive" based on network connectivity, and (3) computation of network-level measures to identify SOIE network characteristics observed among "dead" and "alive" systems. The framework is presented as a flow chart in Figure 1.

SOIE evolution is initiated with a 5-node model, where nodes represent firms and edges between them represent material synergies and/or social ties. The network is then allowed to evolve iteratively over 50 time steps. Every step consists of three decisions: (1) whether nodes should be attached or disconnected, and if yes, how many and to which nodes, (2) whether links should be added or 
removed, and if yes, how many and between which nodes, and (3) is there a large-scale perturbation i.e., "disaster." The evolution simulation was then allowed to repeat for 1000 iterations.

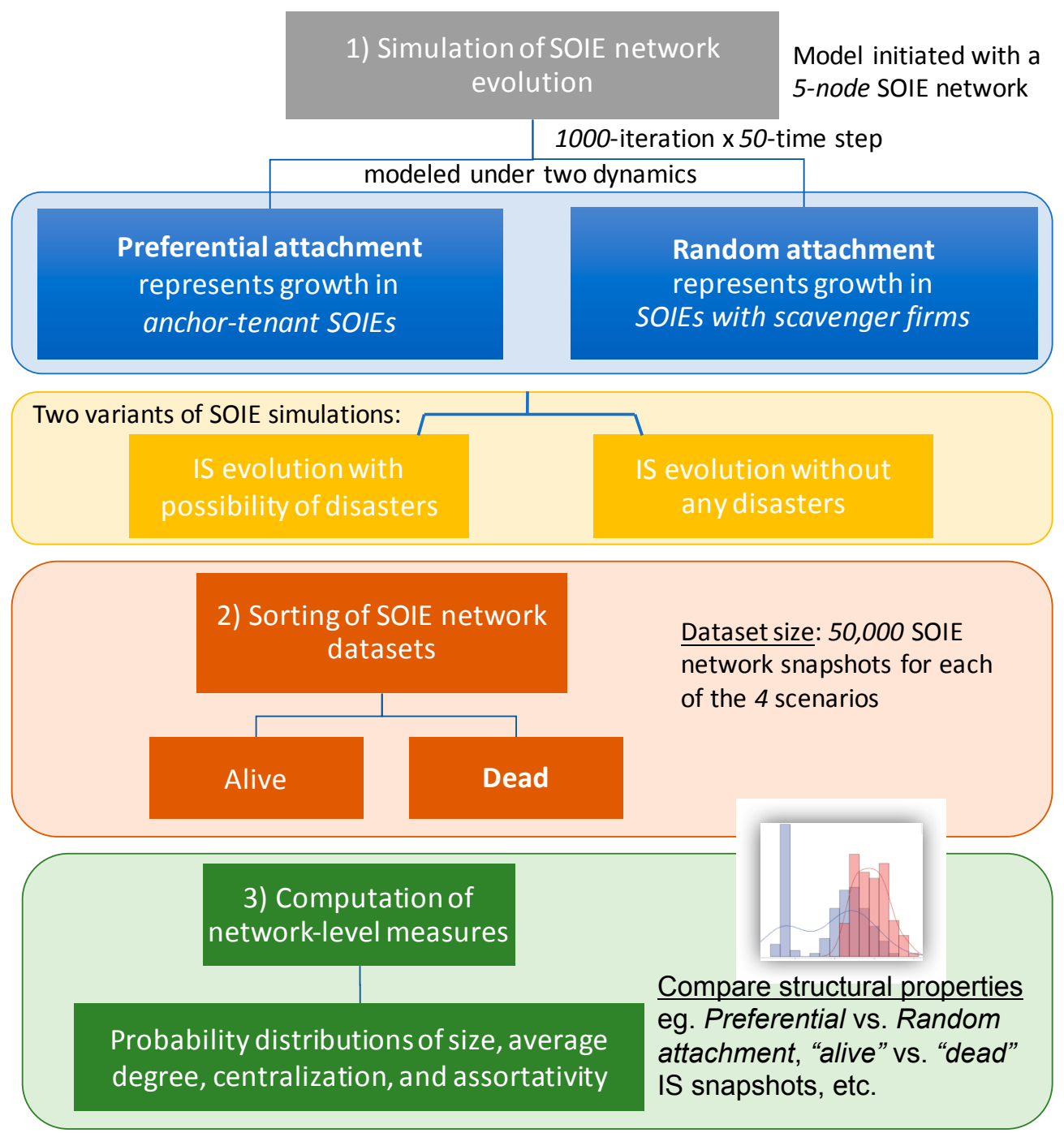

Figure 1. Flow chart presenting the stochastic framework developed to identify structural properties of resilient SOIEs.

This same 5-node model is used to seed every iteration throughout the study to maintain consistency. Each node is first assigned three attributes, 91) social capital denoted by $C_{s}$, (2) natural capital denoted by $C_{n}$, and (3) financial capital, denoted by $C_{f}$, where each is a random number between 0 and 1 . These values are proxies for a firm's endowment with social, environmental and economic attributes that are important antecedents and lubricants in symbiosis development (Table 1). These attributes are assigned to nodes (firms) to govern the evolution of SOIEs through the addition and deletion of firms and synergies, that is nodes with higher values of each of the capitals have a greater probability of persisting in the network over time. A fourth node attribute $\gamma_{\mathrm{i}}$ is also defined as

$$
\gamma_{i}=\frac{\text { Number of connections of node } \mathrm{i}}{\text { Total possible connections in graph } \mathrm{G}}
$$


where $i$ is a node in a graph G. Based on the above-mentioned attributes, a score $S_{i}$ of each node $i$ is defined as

$$
S_{i}=\sqrt{C_{s, i}^{2}+C_{n, i}^{2}+C_{f, i}{ }^{2}+\gamma_{i}^{2}}
$$

The score of a node is used as a measure of its potential to engage in symbiosis, where a high value indicates strong inclination to be in symbiotic relationships.

The network evolution is modeled under two dynamics: (1) preferential attachment that represents growth in anchor-tenant SOIEs and (2) random attachment that represents growth in SOIEs with scavenger firms. First, it is determined whether a new node is to be added or disconnected. This is chosen at random. Under the preferential attachment dynamic, the probability for a new node to be added is $75 \%$, while under the random evolution dynamic the probability of node attachment or disconnection is $50 \%$. This difference in probabilities may be thought of as growth rates, with random networks growing more slowly. Further, in our model, we aim to bring out qualitative trends that mimic the behavior of real-world networks rather than to make exact quantitative predictions. The results are dependent on the decision-making processes involved in the two dynamics, which is explained in detail below. Therefore, the results are not expected to different significantly if the growth rates are changed. The simulations also show that the qualitative trends do not depend in any significant manner on the growth rates.

To determine the site of node attachment or disconnection, a probability $\mathrm{P}_{n, \mathrm{i}}$ is then attached to each existing node in the network. If a new node is to be attached, the probability that a node attaches at site $i, P_{n, i}$ is assigned as $P_{n, i} \propto S_{i}$, i.e., an incoming node takes into account the local attributes of the node as well as the connectedness of the node. If a node is to be disconnected, then the probability $P_{n, i}$ for a node to be disconnected is assigned as $P_{n, i} \propto 1 / S_{n, i}$ i.e., nodes with lower score have a higher chance of being disconnected.

The model also allows for the update, i.e., addition or removal of synergies in a manner similar to the addition and disconnection of nodes. Under preferential attachment, there is a $75 \%$ chance that a link is added, while under random attachment, there is a $50 \%$ chance for addition of synergies. Addition or deletion of synergies between pairs need to take into account collective strength of the pair of nodes under consideration. Therefore, we assign a score $S_{i, j}$ to each pair in the network where

$$
\mathrm{S}_{\mathrm{i}, \mathrm{j}}=0.5 *\left(\mathrm{~S}_{\mathrm{i}}+\mathrm{S}_{\mathrm{j}}\right) \text {. }
$$

A high $S_{\mathrm{ij}}$ indicates that there is a strong potential for the nodes to connect given the high individual $S_{i}$ and $S_{j}$ that takes into account their respective natural, social and financial capital, and their connectedness to the rest of the system. Now probabilities $P_{s, \text { ij }}$ are assigned to every pair $i, j$. Again, if a link is to be added, $P_{s, i j} \propto S_{i j}$, and if synergies are to be removed, then $P_{s, i j} \propto 1 / S_{i j}$. It is possible, that the two nodes selected this way already have a link between them. In this case, these nodes are excluded and we search for the next strongest pair of nodes. This process is repeated until the pair of nodes with the highest $S_{\mathrm{ij}}$ without an existing link is found. Similarly, in the case of link removal, the two nodes selected may not have an existing connection, so a search is conducted to find the link between the weakest two nodes in the system. This link is then removed. Under the random attachment dynamic, every node $i$ and every pair $i$, $j$ have equal $P_{n, i} P_{s, i j}$ regardless of their scores.

This study models an additional scenario for the two growth models: the introduction of external perturbations or "disasters" that cause large-scale deletion of edges and nodes, and that simulate natural disasters or major policy changes that affect some or all of the actors in a network. This scenario is modeled such that there is about 1 in 10 chance that a disaster occurs at each time-step, and the model permits a maximum of two disasters for each simulation in order to give a network enough time to recover. In the event of a disaster, the lower 50th percentile of nodes, ordered according to their respective $S_{i}$, go offline. While these numbers are assigned with some discretion, they were chosen to make sure that there is neither an overabundance of disasters that inhibit IS evolution, nor a paucity of disasters that impede the analysis. There is a certain amount of discretion in the way the percentages 
and the probabilities have been chosen, but the guiding motivation behind setting these probabilities was to ensure that we observe as much variability in behavior as possible in simulations. To maintain consistency, the same evaluation criteria were applied to compare network structural properties of anchor-tenant SOIEs and SOIEs with scavenger firms.

Once the comprehensive data set of $\sim 50,000$ network snapshots is obtained, networks are analyzed at every iteration across all our simulations and sorted into the categories "dead" and "alive." The sorting is based on network size and connectivity. To sort these networks, we test for the presence of IS, defined by having at least one instance of a "3-2" node-edge configuration, i.e., at least three firms engaged in at least two synergies. If the network passes this criterion, it is considered alive. If no instance of such a structure is found, it is declared dead. The dead networks are then listed and analyzed back up to five time steps to understand the cause of death by analyzing the system's route to collapse. This sorting is done separately for both preferential attachment and random growth data sets.

Graph-level measures, such as size, average degree, centralization, and assortativity, are computed for sets of "dead" and "alive" network snapshots to compare structural properties of SOIEs that survive with those that do not. In addition, the characteristics of networks that are "alive" and "dead" are compared in the face of these modeled disasters versus normal operating conditions.

\section{Results}

Table 3 presents the number of "alive" and "dead" networks sorted from the data set of SOIE evolution. As expected, the majority of the SOIE network snapshots were "alive." This is because SOIE systems are expected to grow in terms of number of firms and synergies so long as the economics, regulatory structures and relationship dynamics remain favorable. There is a significant difference in the death rate between networks that grow by preferential attachment $(0.3 \%)$ and those that grow randomly $(7 \%)$. However, when disasters are simulated in the models, the number of "dead" networks more than double. This suggests that the "alive" networks are not necessarily in a resilient state and partnerships and/or firms are unlikely to remain intact if conditions were to change radically due to major external perturbations, such as raw material supply disruption, market decline, and government policy changes.

Table 3. Number of "alive" and "dead" networks collected for topological analysis.

\begin{tabular}{ccc}
\hline Number of SOIE Network Snapshots & Alive IS & Dead IS (\% Dead) \\
\hline Random Growth & 47,803 & $3734(7 \%)$ \\
Preferential Attachment & 49,921 & $193(0.3 \%)$ \\
Random Growth with Disaster & 45,218 & $7052(13 \%)$ \\
Preferential Attachment with Disaster & 49,746 & $516(1 \%)$ \\
\hline
\end{tabular}

\subsection{Life and Death under Normal Conditions}

Figure 2 presents probability distributions of network measures: network size, characteristic path length, and total degree centralization, in order to compare structural properties of "alive" and "dead" SOIEs. Figure $2 \mathrm{a}, \mathrm{c}, \mathrm{e}$ are results for networks that are modeled to evolve through preferential attachment dynamics, and Figure $2 b, d, f$ are results for evolution following dynamics of random growth. 

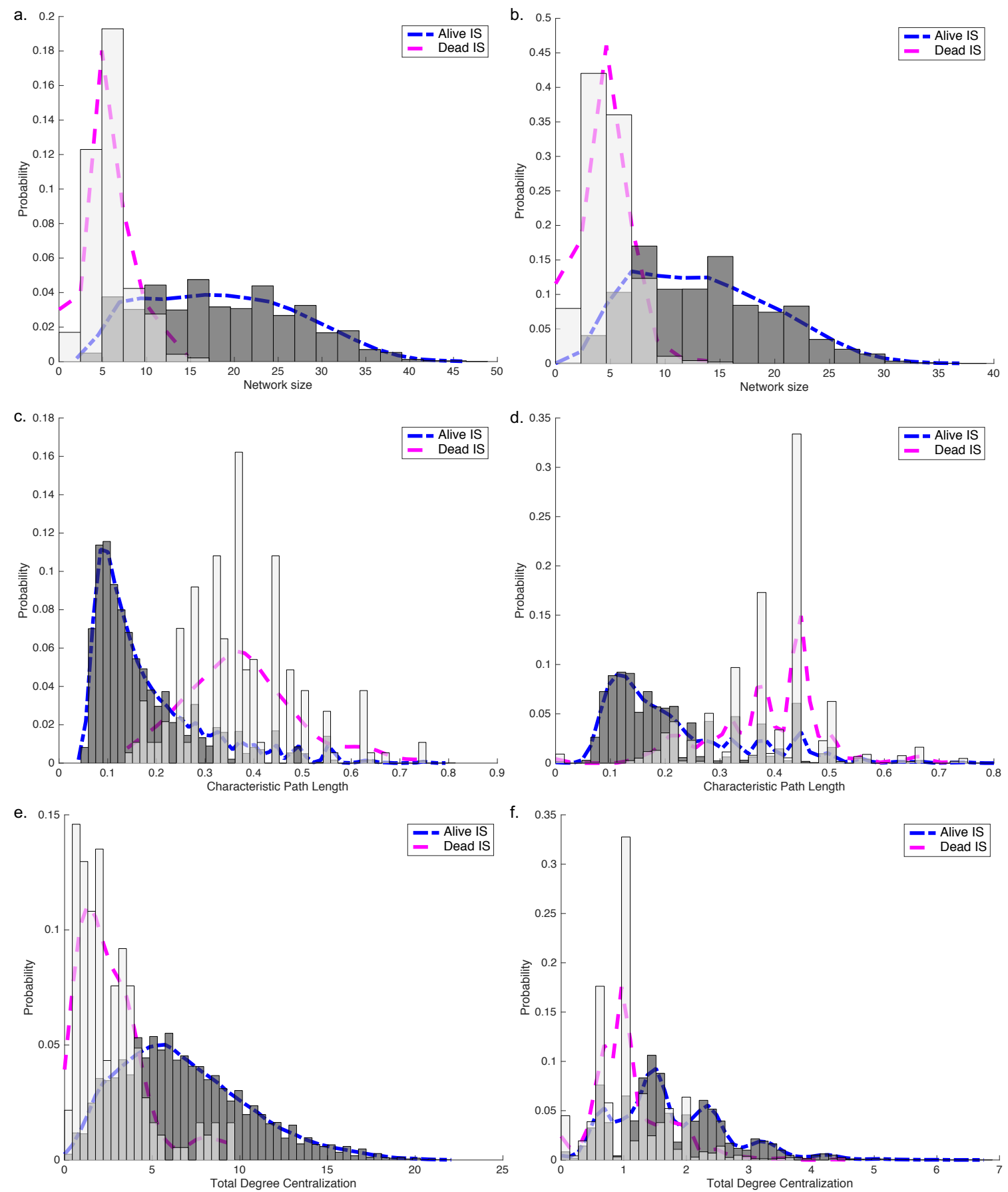

Figure 2. Probability distributions of network measures comparing "alive" and "dead" networks. Sub-plots $(\mathbf{a}, \mathbf{c}, \mathbf{e})$ are modeled to evolve through dynamics of preferential attachment, while (b,d) and (f) are results for SOIEs that evolve in a random fashion.

Probability distributions for network size, i.e., the number of nodes participating in industrial symbiosis, (Figure 2a,b) suggest that smaller SOIEs tend to have a higher probability of being "dead" under either evolution dynamic. However, comparison of the probability distributions of network size for "dead" SOIEs in Figure 2a,b highlights that SOIEs that evolve in a random fashion have a higher probability of being "dead" in the early stages of its formation, especially when networks are smaller. Focusing on the distribution of network size of "alive" SOIEs in Figure 2a,b, it is evident that networks based on random growth dynamics are relatively smaller than those growing based 
on preferential attachment. The probability of finding larger "alive" SOIEs following preferential attachment growth model is evenly distributed in comparison to those growing in a random manner, that is random networks skew smaller in total network size than preferential networks. Figure $2 \mathrm{c}, \mathrm{d}$ plot probability distributions for characteristic path length (CPL), i.e., the average shortest path length between any two nodes in the network, for "dead" and "alive" SOIEs growing through preferential attachment and random growth dynamics. A small CPL suggests the participating firms are well connected, and any two nodes that are not engaged in direct synergies can be easily reached through one of their neighbors. Results show that "alive" networks under both growth scenarios tend to have a smaller CPL in comparison to "dead" networks: that is, they are better connected or clustered. Comparison of probability distributions for the two models of SOIE growth does not demonstrate a sizable difference. However, the probability of finding "dead" networks with larger CPL is relatively higher in the random growth model.

Results for the probability distribution of total degree centralization, i.e., an expression of how tightly the graph is organized around a core node with the greatest number of symbiotic synergies, for "dead" and "alive" SOIEs growing through preferential attachment and random growth dynamics are shown in Figure 2e,f. Greater total degree centralization is observed for "alive" networks in comparison to "dead" ones, and this distinction is very pronounced in networks growing under a preferential attachment paradigm. In addition, looking at the probability distribution for "dead" SOIEs in Figure 2e, two bumps in the curve are observed. The first peak suggests that a high probability of "dead" networks with small total degree centralization (that is the network is too small to have a core). The second smaller bump suggests that a few "dead" SOIE network were found with high total degree centralization, i.e., death can occur even when nodes are well-connected to a core.

\subsection{Life and Death under Disaster Conditions}

Figure 3 presents probability distributions of network density, i.e., the proportion of direct ties in a network relative to the total number possible to compare "alive" and "dead" SOIEs. Figure 3a,c are results for SOIE evolution modeled without considering large-scale disasters, and Figure 3b,d are results for SOIE evolution that allows large-scale disasters. In addition, Figure $3 \mathrm{a}, \mathrm{b}$ are modeled to evolve through preferential attachment dynamics, and Figure $3 c, d$ are results for SOIE evolution following dynamics of random growth. Figure 3 highlights that "alive" networks tend to have a lower density in comparison to "dead" networks. Since "alive" networks tend to be larger than dead networks (as evident from Figure 2a,b), the lower network density is expected. By comparing results for probability distribution in Figure 3a,b, and Figure 3c, d for SOIE growth, it is noted that the inclusion of disasters results in greater probability of finding dead networks with high density, which is due to small network size (also evident from Table 3). 

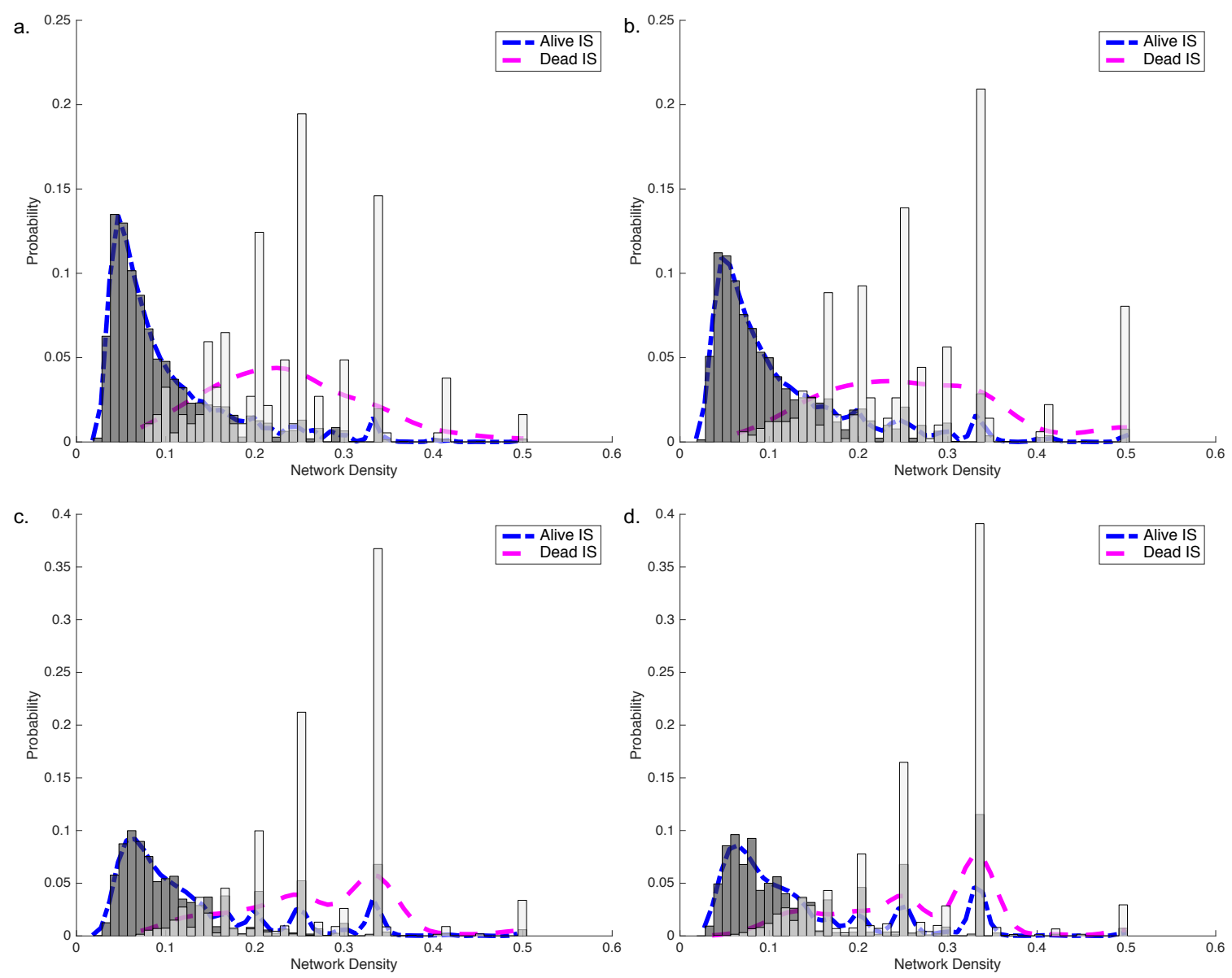

Figure 3. Comparison of "alive" and "dead" networks using probability distributions of network density. Sub-plots $(\mathbf{a}, \mathbf{b})$ are modeled to evolve through dynamics of preferential attachment, while $(\mathbf{c}, \mathbf{d})$ are results for SOIEs that evolve in a random fashion. Sub-plots $(\mathbf{a}, \mathbf{c})$ are results for evolution without large-scale disasters. Sub-plots $(\mathbf{b}, \mathbf{d})$ are results with large-scale disasters.

\subsection{Random and Preferential Attachment Driven SOIE Evolution}

Figure 4 presents probability distributions of network measures,-network degree, assortativity, and number of components-in order to compare structural properties of SOIEs growing by preferential attachment with those growing in a random manner. Figure $4 a, c, e$ are results for "alive" networks and Figure $4 b, d, f$ are results for "dead" networks. In addition, results presented in this figure account for "dead" and "alive" networks due to large-scale disasters. Figure 4a,b presents probability distribution of network degree, the total number of synergies in an SOIE, for "alive" and "dead" networks, respectively. It is evident from probability distributions of network degree for "alive" networks (Figure 4a) that SOIE evolving in a random manner have a higher probability of having fewer synergies in comparison to networks growing with preferential attachment dynamics. A similar trend is observed in the case of "dead" networks (Figure $4 \mathrm{~b}$ ) as well, but in addition, we notice that a majority of random growth "dead" SOIEs have no connections at all. 

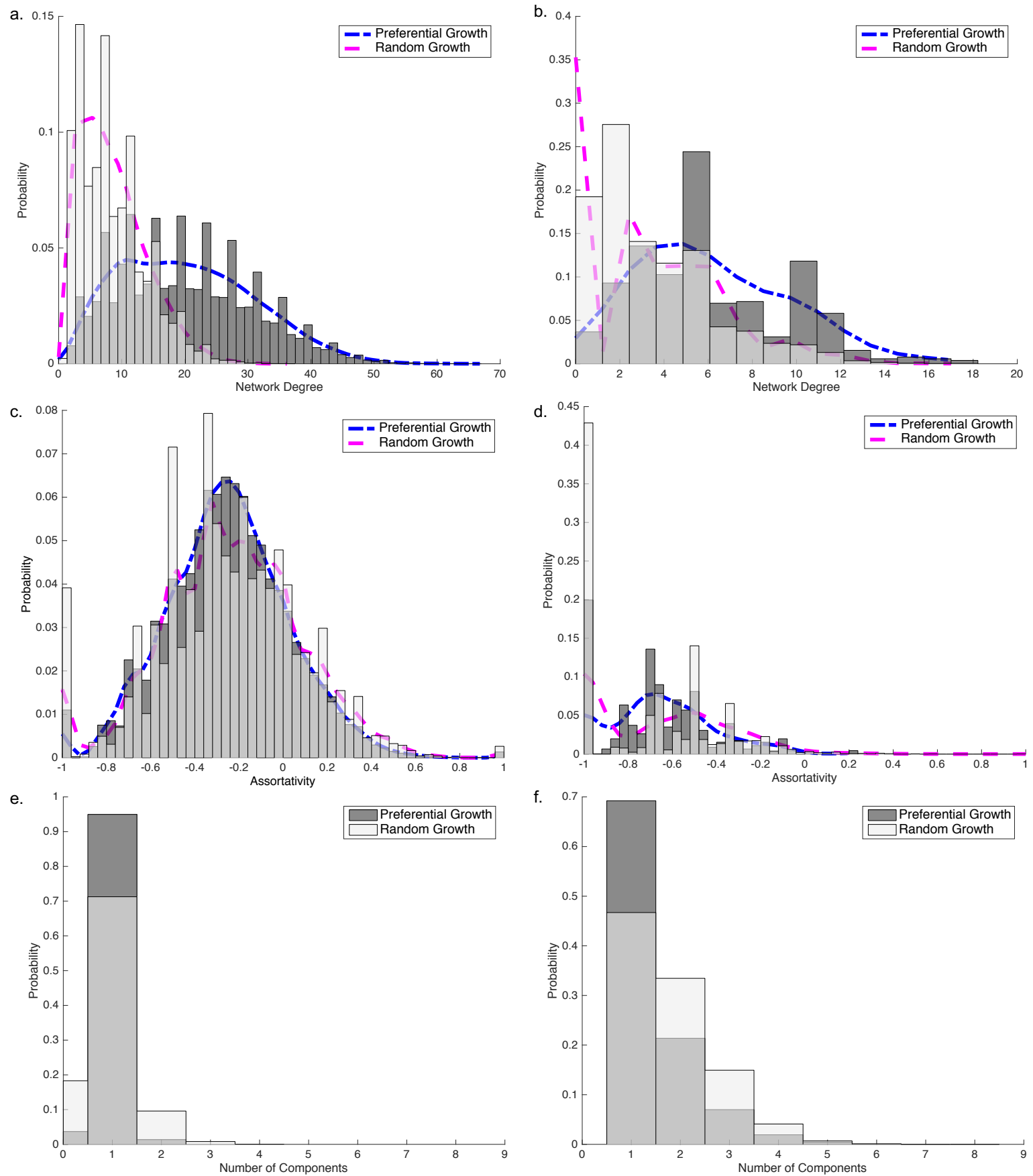

Figure 4. Probability distributions of network measures for comparing SOIE evolution under dynamics of preferential attachment and random growth. Sub-plots $(\mathbf{a}, \mathbf{c}, \mathbf{e})$ are results for "alive" IS, while (b,d,f) are results for "dead" IS. Large-scale disasters are modeled in the simulation of network evolution.

Figure $4 \mathrm{c}, \mathrm{d}$ plot probability distributions for degree assortativity, i.e., the tendency of firms to associate and develop synergies with similar nodes in terms of their degree or connectedness, for "alive" and "dead" SOIEs. A degree assortative network is one that links highly connected firms to other high degree nodes, while degree disassortative networks have connections between highly connected firms and ones with low connectivity. A measure of assortativity range from -1 (completely disassortative) to 1 (completely assortative). Assortativity results for "alive" networks (Figure 4c) showcase a higher probability of successful SOIEs to be disassortative under both growth scenarios. 
However, the probability distribution of assortativity for "dead" networks (Figure 4d) suggest that failed SOIEs tend to be more disassortative than systems that survive. This is partly observed due to the smaller network size of dead networks. However, this result also suggests that if a high degree firm drops out of a disassortative SOIE, it has a great impact on the overall connectedness of the network.

Figure $4 \mathrm{e}, \mathrm{f}$ highlight the distribution of components, i.e., the number of sub-graphs that are disconnected from each other, for "alive" and "dead" SOIEs. Focusing on the alive networks (Figure 4e), it is noted that while SOIEs following preferential attachment dynamics have a higher probability of having a single component, networks growing in a random fashion have a higher probability of having more than one component. This trend is also observed in the case of "dead" networks from Figure $4 \mathrm{f}$.

\section{Discussion}

We have developed a stochastic approach to understanding the network properties of self-organized industrial ecosystems that allow them to be "alive" and result in their "death". The approach takes an iterative process that on completion enables thorough quantification and definition of the problem space with respect to topological attributes that result in the collapse of IS. This study serves as a demonstration of the feasibility of a data-driven methodology to identify structural properties that influence the resilience of SOIE networks growing through different dynamics.

Analysis of probability distributions highlight the network properties associated with "dead" and "alive" networks for both preferential and random growth dynamics. Based on results from Figure 2a,b, we observe that smaller SOIEs tend to have a higher probability of being "dead," which suggests that SOIE networks are at their most vulnerable during the early stages of inception. Individual firms in an industrial ecosystem begin developing synergies among themselves and develop more numerous relationships and deeper social ties with partners as the system ages. Since the likelihood of survival for an SOIE increases drastically with size, greater efforts are needed to mitigate risk at the early stages by managers and participating firms to avoid network collapse once they become aware of the network. Moreover, probability distributions of CPL (Figure 2c,d) show that "alive" networks tend to have smaller CPL than "dead" networks, which implies that "alive" networks are more cohesive since the distance between participating firms is low. This structural property also influences the ability of any pair of nodes to communicate with each other, which in turn contributes to its resilience. Large external perturbations or disasters force more networks to collapse under in either growth dynamic, particularly when networks are smaller, more disassortative, and have fewer components.

These results support the argument that a real structural shift occurs after symbiotic interactions are identified and inter-firm communications are institutionalized, such that more firms communicate directly or through established institutions, such as the Symbiosis Institute in Kalundborg or the Kwinana Industries Council. Institutionalization enables shared learning, expansion, diversification of connectedness, and building of adaptive capacity and learning among the agents in the system [17]. It is expected that some interactions that were viable at one time may cease when they no longer make financial or regulatory sense, or that some firms may go out of business. Our model accounts for this ongoing dynamic by adding and deleting synergies probabilistically in each time step. If the relationships among members of the network are cemented in a sense of community and willingness to cooperate, i.e., high levels of social capital, then firms will continue seeking out opportunities for collaboration and sustainable resource management. We also find that "alive" networks tend to have relatively higher total degree centralization than "dead" ones (Figure 2e,f), thus suggesting that most successful networks evolve around a few critical firms. However, results also point out that beyond a certain point, highly centralized firms become sources of vulnerabilities for the network. Therefore, it is important to avoid development of SOIE networks with a single critical node, as was the case in Barceloneta. Notably, mature structures for both preferential and random growth appear to have similar structures: a large number of nodes, ties, and multiple components. These results imply that resilience can be low in the early stages of system development when the various industrial actors 
are new to the system and not deeply socially embedded in it, and that in later stages the level of social capital/connectivity among actors may be so strong that it inhibits innovative responses to change [18].

In addition, the stochastic approach was able to compare structural properties for SOIEs growing through preferential attachment and random growth dynamics. Results (Figure 4a,b) suggest that SOIEs evolving randomly tend to have fewer synergies in comparison to networks growing with preferential attachment dynamics. Also, unlike SOIEs growing through random dynamics, network degree is more evenly distributed for SOIEs growing through preferential attachment. These results imply that networks evolving with preferential attachment dynamics are more stable and likely to survive when they have more by-product and utility synergies, but randomly growing SOIE networks are more stable and likely to survive with relatively fewer synergies. This supports the hypothesis that many small IS networks exist without awareness of partaking in symbiosis. Results for degree assortativity (Figure 4c) indicate that "alive" networks tend to be slightly disassortative for both growth dynamics, which implies critical firms, in terms of the number of synergies, tend to form new synergies with relatively less critical firms. However, as noted in Figure 4d, "dead" networks are far more disassortative, suggesting that greater deliberation is required when adding new synergies (especially utility sharing) to avoid over-concentration of vulnerability at a few firms in the SOIE. Additionally, on comparing the number of components in networks, SOIEs evolving randomly are found to have more than one component, while preferential attachment dynamics resulted in a single component or cluster in the network. SOIE networks growing in a random fashion tend to fracture and form smaller sub-graphs more often than in the case of preferential attachment dynamics for SOIE evolution.

\section{Conclusions}

Unplanned, self-organized industrial ecosystems provide multiple social, environmental, and economic benefits to a region such as increased industrial activity and employment, reduced industrial emissions, etc., which means multiple stakeholders (industry owners, regional policy-makers, contractors, local community, and environmental agencies, among others) stand to gain from the successful genesis, planning, and implementation of these systems. However, these stakeholders also share the responsibility to maintain conditions conducive for such multifunctional SOIEs to thrive, after symbiosis is uncovered. For this reason, it is imperative to have adequate information about structural properties of SOIEs for making critical decisions to build resilience, which has been difficult to obtain because of the paucity of data on SOIEs. In addition, whatever little information is available is mostly based on individual case studies of industrial symbiosis rather than a broad sample of networks. Case study approaches to understand the resilience of IS provide useful insights, but their utility is limited since they are inadequate for understanding properties that may cause SOIEs to collapse or survive, and for comparing advantages and disadvantages of different SOIE growth dynamics.

To address this concern, this study's data-driven approach develops large datasets of SOIE evolution for quantitative assessment and identification of network characteristics that play a role in "life" and "death" of SOIEs. Insights from this approach are more generalizable and allow researchers to prepare contingency plans for SOIEs for proactive disaster preparedness and response, such as by encouraging redundant linkages and multiple clusters or components rather than single core. While the approach presented in this study is mostly focused on the structural aspects of self-organized industrial ecosystems, the ability to maintain the functionality is also an important aspect of building the resilience of any complex system. However, it is important to note that structural integrity is especially crucial for maintaining functionality in SOIEs, thus the resilience of these systems is especially sensitive to its structural robustness. This study represents the first step and warrants more research into the structural dynamics of SOIEs. Additionally, SOIEs represent only one type of industrial symbiosis dynamic as identified in by Boons [4]. In the future, modeling of the other six 
types could reveal similarities and differences in how the different types of networks develop under normal conditions and with external perturbations.

Acknowledgments: The authors wish to thank Marian Chertow for her suggestions in developing the manuscript; she and W.A. previously developed an early version of this topic. They also thank the anonymous reviewers for their suggestions to strengthen the manuscript. Funding for open access publication was provided by the Illinois Institute of Technology Stuart School of Business.

Author Contributions: W.A. and S.C. conceived the paper and designed the modeling framework; S.C. and R.K. developed the model and analyzed the data; W.A. and S.C. wrote the paper.

Conflicts of Interest: The authors declare no conflict of interest.

\section{References}

1. Ehrenfeld, J.; Gertler, N. Industrial Ecology in Practice: The Evolution of Interdependence at Kalundborg. J. Ind. Ecol. 1997, 1, 67-79. [CrossRef]

2. Jacobsen, N.; Anderberg, S. Understanding the Evolution of Industrial Symbiotic Networks: The Case of Kalundborg. In Economics of Industrial Ecology: Materials, Structural Change, and Spatial Scales; van den Bergh, J.C.J.M., Janssen, M., Eds.; MIT Press: Cambridge, MA, USA, 2005.

3. Chertow, M.R. “Uncovering” Industrial Symbiosis. J. Ind. Ecol. 2007, 11, 11-30. [CrossRef]

4. Boons, F.; Chertow, M.; Park, J.; Spekkink, W.; Shi, H. Industrial Symbiosis Dynamics and the Problem of Equivalence: Proposal for a Comparative Framework. J. Ind. Ecol. 2016. [CrossRef]

5. Gibbs, D.; Deutz, P. Reflections on implementing industrial ecology through eco-industrial park development. J. Clean. Prod. 2007, 15, 1683-1695. [CrossRef]

6. Ashton, W. The Structure, Function and Evolution of a Regional Industrial Ecosystem. J. Ind. Ecol. 2009, 13, 228-246. [CrossRef]

7. Holling, C.S. Understanding the Complexity of Economic, Ecological, and Social Systems. Ecosystems 2001, 4, 390-405. [CrossRef]

8. Zhu, J.; Ruth, M. Exploring the resilience of industrial ecosystems. J. Environ. Manag. 2013, 122, 65-75. [CrossRef] [PubMed]

9. Chopra, S.S.; Khanna, V. Understanding resilience in industrial symbiosis networks: Insights from network analysis. J. Environ. Manag. 2014, 141, 86-94. [CrossRef] [PubMed]

10. Yap, N.T.; Devlin, J.F. Explaining Industrial Symbiosis Emergence, Development, and Disruption: A Multilevel Analytical Framework. J. Ind. Ecol. 2016, 21, 6-15. [CrossRef]

11. Fraccascia, L.; Giannoccaro, I.; Albino, V. Rethinking Resilience in Industrial Symbiosis: Conceptualization and Measurements. Ecol. Econ. 2017, 137, 148-162. [CrossRef]

12. Walls, J.L.; Paquin, R.L. Organizational Perspectives of Industrial Symbiosis: A Review and Synthesis. Organ. Environ. 2015, 28, 32-53. [CrossRef]

13. Van Beers, D.; Bossilkov, A.; Corder, G.; van Berkel, R. Industrial Symbiosis in the Australian Minerals Industry. J. Ind. Ecol. 2007, 11, 55-72. [CrossRef]

14. Schwarz, E.J.; Steininger, K.W. Implementing nature's lesson: The industrial recycling network enhancing regional development. J. Clean. Prod. 1997, 5, 47-56. [CrossRef]

15. Ashton, W.S.; Bain, A. Assessing the "Short Mental Distance" in Eco-Industrial Networks. J. Ind. Ecol. 2012, 16, 70-82. [CrossRef]

16. Chertow, M.R.; Ashton, W.S. The social embeddedness of industrial symbiosis linkages in Puerto Rican industrial regions. In The Social Embeddedness of Industrial Ecology; Boons, F., Howard-Grenville, J., Eds.; Edward Elgar: Cheltenham, UK; Northampton, MA, USA, 2009; pp. 128-151.

17. Baas, L.W.; Boons, F.A. An industrial ecology project in practice: Exploring the boundaries of decision-making levels in regional industrial systems. J. Clean. Prod. 2004, 12, 1073-1085. [CrossRef]

18. Granovetter, M. Economic Action and Social Structure: The Problem of Embeddedness. Am. J. Sociol. 1985, 91, 481-510. [CrossRef] 